\title{
Does Robotic Distal Gastrectomy Facilitate Minimally Invasive Surgery for Gastric Cancer?
}

\author{
TAKESHI KUBOTA ${ }^{1}$, DAISUKE ICHIKAWA ${ }^{1,2}$, TOSHIYUKI KOSUGA ${ }^{1}$, \\ KATSUTOSHI SHODA ${ }^{1}$, SHUHEI KOMATSU ${ }^{1}$, HIROTAKA KONISHI ${ }^{1}$, \\ ATSUSHI SHIOZAKI ${ }^{1}$, HITOSHI FUJIWARA ${ }^{1}$, KAZUMA OKAMOTO $^{1}$ and EIGO OTSUJI ${ }^{1}$ \\ ${ }^{1}$ Division of Digestive Surgery, Department of Surgery, Kyoto Prefectural University of Medicine, Kyoto, Japan; \\ ${ }^{2}$ Department of Surgery 1, Yamanashi University, Yamanashi, Japan
}

\begin{abstract}
Background: Robotic surgical systems have advantages over laparoscopic surgery. The object of this study was to assess patients' postoperative outcomes after robotic distal gastrectomy (RDG) compared to laparoscopic distal gastrectomy (LDG). Patients and Methods: We retrospectively evaluated 21 and 119 patients who underwent $R D G$ and $L D G$, respectively, for stage I gastric cancer. Short-term outcomes were compared including drain amylase levels and quality of life using a post-gastrectomy syndrome questionnaire. Results: No patients experienced pancreatic fistula or anastomosis-related complications following RDG. The rate of overall complications tended to be lower in patients undergoing RDG. In addition to drain amylase level, inflammatory findings were lower after RDG than LDG. Patients undergoing RDG experienced a better quality of life and less dissatisfaction in daily life. Conclusion: RDG is feasible and produced highlysatisfactory results for early gastric cancer. We believe that the effective use of robotic surgery will increase safety and precision in minimally invasive surgery.
\end{abstract}

Laparoscopic surgery has contributed to progress in gastric cancer surgery since it was first used for this type of cancer in the 1990s. Developments in surgical instruments, the establishment of well-organized and standardized procedures, and a deeper understanding of local anatomy have reduced operative time, blood loss, and postoperative

Correspondence to: Takeshi Kubota, MD, Ph.D., Division of Digestive Surgery, Department of Surgery, Kyoto Prefectural University of Medicine, 465 Kajii-cho, Kawaramachi-Hirokoji, Kamigyo-ku, Kyoto 602-8566 Japan. Tel: +81 752515527, Fax: +81 752515522, e-mail: tkubot@koto.kpu-m.ac.jp

Key Words: Gastric cancer, robotic distal gastrectomy, pancreatic fistula, minimally invasive surgery, quality of life. complications, which results in earlier recovery and shorter hospital stays $(1,2)$. Use of laparoscopic surgery has spread quickly and widely in response to increased demand for the technique, and minimally invasive surgery is now a trend in gastric cancer surgery.

However, postoperative complications following laparoscopic distal gastrectomy (LDG) are a concern. In particular, postoperative pancreatic fistula can be fatal because it may result in intra-abdominal abscess, secondary anastomotic leakage, and severe bleeding. A multiinstitutional phase II trial which assessed the safety of LDG for clinical stage I gastric cancer showed that the incidence of pancreatic fistula was as low as $1.1 \%$ (3). However, an analysis of a Japanese national clinical database by Hiki et $a l$. reported that the rate of pancreatic fistula after LDG was higher compared to open distal gastrectomy (2.2\% vs. $1.0 \%$; $p=0.04)$ (4). This difference suggests that the results of clinical trials performed in limited high-volume centers may not be applicable to general hospitals. Ida et al. stated that one of the mechanisms contributing to postoperative pancreatic fistula is excess compression by the straight forceps used in LDG, which can cause blunt pancreatic injury and secondary leakage of pancreatic juice (5).

Robotic surgical systems have advantages over laparoscopic surgery, and fewer complications and higher quality surgery are expected. The da Vinci robotic surgical system (Intuitive Surgical, Sunnyvale, CA, USA) provides a three-dimensional high-definition, magnified view of the operating field, consists of flexible instruments, and involves tremor filtering and motion scaling, which enable surgeons to overcome some of the limitations of laparoscopic surgery $(6,7)$. These characteristics are particularly important in gastric cancer surgery because of the need for precise lymph node dissection and good operating view. Initial studies have demonstrated the safety and efficacy of robotic distal gastrectomy (RDG) for gastric cancer, indicating less blood loss and shorter hospital stays compared with LDG (8-11). In a multi-institutional 
prospective study, Uyama et al. suggested RDG resultes in a lower postoperative pancreatic fistula rate (12). Consequently, the effective use of robotic surgical systems for gastric cancer can avoid excessive compression of the pancreas during suprapancreatic lymph node dissection, which reduces the postoperative pancreatic fistula rate.

In this study, we investigated whether a robotic approach facilitated minimally invasive surgery by comparing shortterm outcomes and quality of life (QOL) following RDG compared with LDG.

\section{Patients and Methods}

Patients. From February 2015 to February 2019, LDG or RDG with D1 or D1+ lymph node dissection was performed in 149 patients with clinical stage I gastric cancer at our hospital. Patients with a history of open laparotomy, combined resection of other organs excluding cholecystectomy, other organ malignancies, and residual gastric cancer were excluded from the study. Twenty-one patients agreed to robotic surgery and underwent RDG, while LDG was performed in 119 patients. Patients' clinical and pathological stages were determined based on the 14th Edition of the Japanese Classification of Gastric Carcinoma (13). This study conformed to the ethical guidelines of the World Medical Association's Declaration of Helsinki. All patients provided written informed consent for surgery and the use of their clinical data as required by the Institutional Review Board of the Kyoto Prefectural University of Medicine. This study was performed according to the Recommendations for the Conduct, Reporting, Editing and Publication of Scholarly Work in Medical Journal (14).

Surgeon background. All procedures in this study were performed by two surgeons (TK and DI) who were certified as laparoscopic gastrectomy surgeons by the Japan Society of Endoscopic Surgery and had who undergone the designated robotic surgery training.

Surgical procedures. The da Vinci Si surgical system (Intuitive Surgical) was used for all RDG procedures. All gastrectomies and lymph node dissections were performed based on the Japanese Gastric Cancer Treatment Guidelines (v. 3) (15). RDG was performed by a single surgeon with an assistant only when necessary. Two assistants focused on the interference between the da Vinci arms and the patient's upper arm, iliac crest, and abdominal wall. In LDG, to facilitate a good operating view of the suprapancreatic area, an assistant gently compressed the pancreas using a small gauze when needed. The reconstruction method was determined by the surgeon's experience: Billroth I or Roux-en Y gastrojejunal anastomosis

Perioperative management. All patients underwent preoperative physical examinations, namely laboratory tests, chest and abdominal $\mathrm{X}$-rays, electrocardiogram, and lung function tests. Prophylactic antibiotics were routinely administered $30 \mathrm{~min}$ before surgery and every $3 \mathrm{~h}$ during surgery. Blood biochemical examinations were performed 1,3, and 7 days after surgery, and the drain amylase level was measured 1 and 3 days after surgery. Patients were discharged once it was confirmed that they experienced no postoperative problems such as eating difficulties, blood biochemical abnormalities, or postoperative complications.
Table I. Patient characteristics.

\begin{tabular}{lccr}
\hline & $\begin{array}{c}\text { RDG } \\
(\mathrm{n}=21)\end{array}$ & $\begin{array}{c}\text { LDG } \\
(\mathrm{n}=119)\end{array}$ & $p$-Value \\
\hline Age, years & 59.8 & 67.0 & 0.0268 \\
Sex, male/female & $11 / 10$ & $72 / 47$ & 0.6463 \\
Body mass index $\left(\mathrm{kg} / \mathrm{m}^{2}\right)$ & 20.9 & 22.3 & 0.1067 \\
Tumor diameter, mean $(\mathrm{mm})$ & 23.9 & 34.2 & 0.0339 \\
Pathological T factor, T1/2/3 & $18 / 1 / 2$ & $93 / 20 / 6$ & 0.1606 \\
Pathological N factor, N0/1/2/3 & $19 / 1 / 1 / 0$ & $101 / 9 / 4 / 5$ & 0.3973 \\
Pathological stage, I/II/III & $18 / 3 / 0$ & $104 / 9 / 6$ & 0.2783 \\
Operative time, min & 423.8 & 315.6 & $<0.0001$ \\
Bleeding, g & 21.3 & 51.3 & 0.0223 \\
Lymph nodes harvested, $\mathrm{n}$ & 42.9 & 33.9 & 0.1270 \\
Complication, total*, $\mathrm{n} \%)$ & $1(6.7 \%)$ & $28(24.3 \%)$ & 0.1503 \\
Anastomotic leakage, $\mathrm{n}$ & 0 & 1 & \\
Pancreatic fistula, $\mathrm{n}$ & 0 & 2 & \\
Postoperative bleeding, $\mathrm{n}$ & 0 & 1 & \\
Surgical site infection, $\mathrm{n}$ & 0 & 2 & \\
Intra-abdominal abscess, $\mathrm{n}$ & 0 & 0 & \\
Liver dysfunction, $\mathrm{n}$ & $11^{* *}$ & 6 & \\
Ileus, $\mathrm{n}$ & 0 & 1 & \\
Pneumonia, n & 0 & 1 & \\
Other, n & 0 & 14 & \\
Postoperative hospital stay, days & 11.2 & 12.8 & 0.7215 \\
\hline
\end{tabular}

Continuous variables are presented as means. *Clavien-Dindo classification; ** grade 4 a.

Evaluation of perioperative outcomes. Patient characteristics; operative outcomes (i.e. operative time, blood loss, number of harvested lymph nodes); short-term postoperative outcomes (i.e. complications, drain amylase level); inflammatory findings [i.e. body temperature (BT), white blood cell (WBC) count, or Creactive protein (CRP)]; and length of postoperative hospital stay were evaluated retrospectively and compared between patients undergoing RDG and those undergoing LDG.

The Clavien-Dindo classification system was used to evaluate the severity of postoperative complications for each patient $(16,17)$. The complications assessed in our study were: Anastomotic leakage, pancreatic fistula, postoperative bleeding, surgical site infection, intra-abdominal abscess, liver dysfunction, ileus, and pneumonia within 30 days after surgery. When a patient had multiple complications, the most serious complication was used for grading.

In order to assess postgastrectomy syndrome and QOL, we used the Postgastrectomy Syndrome Assessment Scale 37-item questionnaire (PGSAS-37) developed by the voluntary group, the Japan Postgastrectomy Syndrome Working Party (18). The main outcome measures of the PGSAS-37 are composed of seven symptom scales: Esophageal reflux, abdominal pain, meal-related distress, indigestion, diarrhea, constipation, and dumping syndrome; four independent living status scales: Ingested amount of food per meal, necessity for additional food, quality of ingestion, and ability to work; and one QOL scale: Dissatisfaction in daily life. The total symptom score was calculated by averaging the seven symptom scales. High scores for the ingested amount of food per meal and quality of ingestion indicated favorable outcomes, whilst low scores for seven symptom subscales, total symptom score, the necessity for 


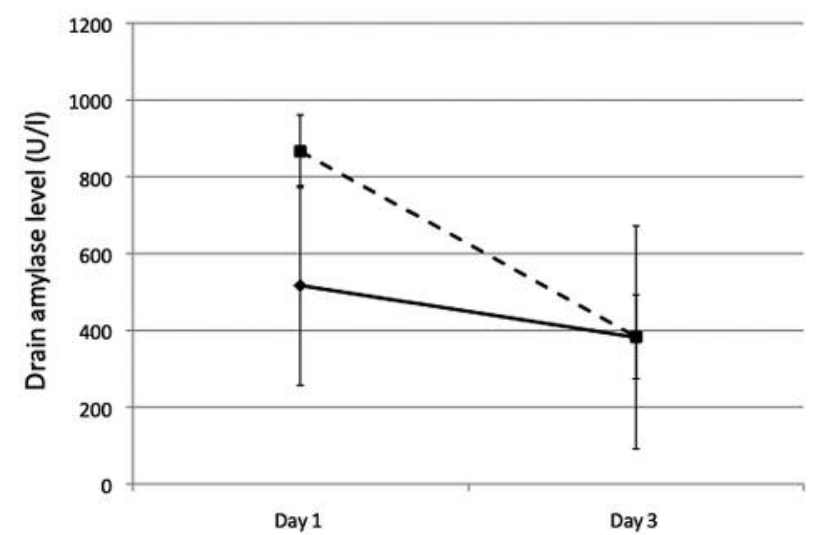

Figure 1. Drain amylase levels on postoperative days 1 and 3 after robotic distal gastrectomy $(R D G)$. Drain amylase levels following $R D G$ were lower than those following laparoscopic distal gastrectomy $(L D G)$ on postoperative day 1. Solid line: RDG; dashed line: $L D G$.

additional food, ability to work, and dissatisfaction in daily life indicated favorable outcomes. The questionnaire was distributed to all participating patients, who were instructed to complete the questionnaire from the time of discharge to 1 month after the procedure and then return the questionnaire to our Department.

Statistical analysis. All statistical analyses were performed using JMP software (v. 13; SAS Institute, Cary, NC, USA). Continuous variables are presented as means and were analyzed using Student's $t$-test or the Mann-Whitney $U$-test, while categorical variables were analyzed using Pearson's chi-squared test. Statistical tests were two-sided, and $p$-values less than 0.05 were considered statistically significant.

\section{Results}

Patient demographics and surgical outcomes. Twenty-one patients underwent RDG (11 men and 10 women) and 119 patients underwent LDG (72 men and 47 women) (Table I). The two groups had similar demographics and clinicopathological characteristics (sex, body mass index, and pathological stage), but the RDG group was comparatively younger. The RDG group had significantly longer operative times (423.8 vs. $315.6 \mathrm{~min} ; p<0.0001$ ), but significantly less blood loss (21.3 g vs. $51.3 \mathrm{~g} ; p<0.0223)$. The mean number of harvested lymph nodes tended to be greater in the RDG group (42.9 vs. 33.9; $p=0.1270$ ), but this difference was not significant. There was no significant difference in length of postoperative hospital stay between the two groups (11.2 vs. 12.8 days; $p=0.7215)$.

Postoperative complications. Pancreatic fistula and anastomosisrelated complications were not observed following RDG. Postoperative complications of Clavien-Dindo grade 2 or more were reported in one patient $(6.7 \%)$ after RDG and in 28 patients
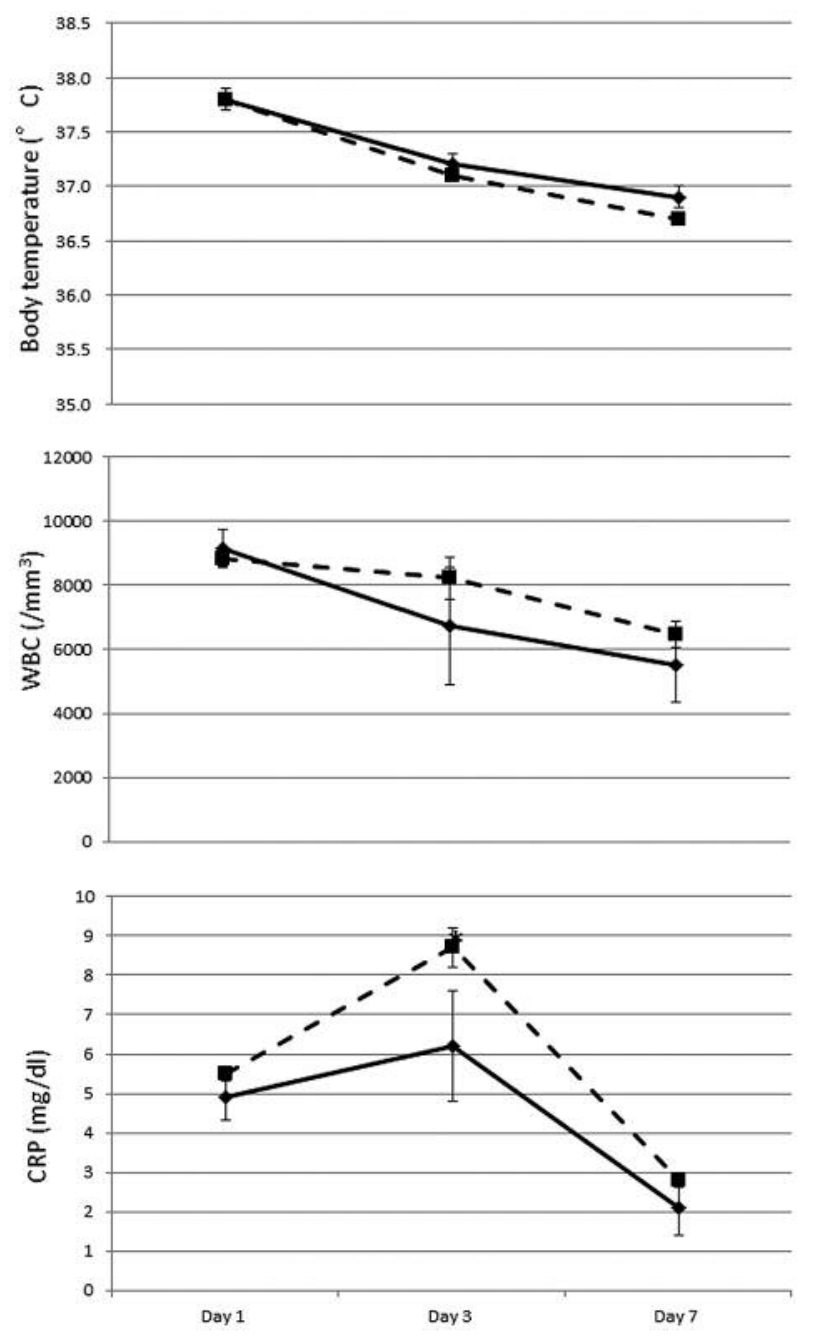

Figure 2. Inflammatory findings of body temperature, white blood cell $(W B C)$ count, and C-reactive protein (CRP) level on postoperative days 1,3 , and 7 after robotic distal gastrectomy (RDG) or laparoscopic distal gastrectomy (LDG). WBC counts and CRP levels were lower after $R D G$ compared with after $L D G$. There was no difference in body temperature between the RDG and $L D G$ groups. Solid line: $R D G$; dashed line: $L D G . *$ Significantly different at $p<0.05$.

(24.3\%) after LDG ( $p=0.1503)$; the complication rates tended to be lower in patients undergoing RDG (Table I). We measured the drain amylase level on postoperative days 1 and 3 to evaluate pancreatic juice leakage (Figure 1). The mean level following RDG was lower than that following LDG on postoperative day 1 (536 U/I vs. $898 \mathrm{U} / 1, p=0.1692)$, but then they decreased and were similar in the two groups on postoperative day 3 .

Inflammatory findings. WBC, CRP, and BT on postoperative days 1, 3, and 7 are shown in Figure 2. The WBC and BT in both groups increased on postoperative day 1 compared with 
Table II. Scores for Post-gastrectomy Syndrome Assessment Scale 37-item questionnaire 1 month postoperatively.

\begin{tabular}{|c|c|c|c|c|c|c|}
\hline \multirow[t]{2}{*}{ Domains } & \multirow[t]{2}{*}{ Main outcome measures } & \multicolumn{2}{|c|}{ RDG } & \multicolumn{2}{|c|}{ LDG } & \multirow[t]{2}{*}{$p$-Value } \\
\hline & & Mean & SD & Mean & $\mathrm{SD}$ & \\
\hline \multirow[t]{8}{*}{ Symptoms } & Esophageal reflux & 1.65 & 1.15 & 2.77 & 1.51 & 0.0331 \\
\hline & Abdominal pain & 1.97 & 1.14 & 2.62 & 1.27 & 0.1557 \\
\hline & Meal-related distress & 2.37 & 0.49 & 2.92 & 0.27 & 0.3314 \\
\hline & Indigestion & 1.85 & 0.95 & 2.03 & 1.72 & 0.7538 \\
\hline & Diarrhea & 1.77 & 1.26 & 1.99 & 1.41 & 0.6560 \\
\hline & Constipation & 2.33 & 1.22 & 2.78 & 1.49 & 0.3957 \\
\hline & Dumping & 1.07 & 0.56 & 1.11 & 1.49 & 0.9275 \\
\hline & Total symptom score & 1.88 & 0.84 & 2.29 & 0.78 & 0.1615 \\
\hline \multirow[t]{4}{*}{ Living status } & Ingested amount of food per meal & 4.11 & 1.76 & 2.54 & 1.88 & 0.0303 \\
\hline & Necessity for additional food & 2.00 & 0.67 & 2.21 & 1.08 & 0.5624 \\
\hline & Quality of ingestion & 2.83 & 1.39 & 2.23 & 1.43 & 0.2482 \\
\hline & Ability for working & 2.50 & 1.43 & 2.61 & 1.50 & 0.8441 \\
\hline QOL & Dissatisfaction with daily life & 2.30 & 1.29 & 3.75 & 2.10 & 0.0559 \\
\hline
\end{tabular}

RDG: Robotic distal gastrectomy; LDG: laparoscopic distal gastrectomy; QOL: quality of life.

preoperative values, then gradually decreased to within normal limits on postoperative day 7 , while the CRP level peaked on postoperative day 3 and decreased thereafter. Overall, the WBC and CRP levels were lower after RDG compared with after LDG. There were no differences in BT between the RDG and LDG groups.

Post-gastrectomy syndrome and QOL. PGSAS-37 scores 1 month postoperatively are listed in Table II. The esophageal reflux score in the PGSAS-37 was significantly lower in the RDG group compared with the LDG group (1.65 vs. 2.77, respectively; $p=0.0331$ ), and the total symptom score tended to be lower for the RDG group compared with the LDG group (1.88 vs. 2.29, respectively; $p=0.1615)$. The ingested amount of food per meal, quality of ingestion, ability to work, and dissatisfaction in daily life were important factors in patient QOL, and the scores for these factors showed that patients undergoing RDG experienced better QOL and less dissatisfaction in their daily lives compared with patients undergoing LDG (2.30 vs. 3.75, respectively; $p=0.0559)$.

\section{Discussion}

Gastric cancer is one of the most common types of cancer worldwide (19), and radical gastrectomy with regional lymph node dissection remains the primary method for treating gastric cancer. In Japan, the demand for less-invasive surgery has recently increased dramatically because the diagnostic rate for early gastric cancer has exceeded $50 \%$ (20). After Kitano et al. reported the first patient undergoing LDG in 1994, Japanese surgeons evaluated this approach as a lessinvasive means of surgery and quickly adopted the technique
(21). According to a survey by the Japan Society for Endoscopic Surgery, more than 10,000 patients underwent distal gastrectomy in 2017, with more than half of these undergoing LDG (22). Robotic surgery for gastric cancer is expected to be even less invasive than LDG; the effectiveness of RDG as a minimally invasive surgery has been verified as being equal to that of LDG. Suda et al. suggested that RDG reduced surgery-related complications, leading to further improvement in short-term postoperative outcomes (23). However, the higher costs and longer operative time associated with RDG might conceivably reduce surgeons' desire to implement RDG. In addition, the results of a prospective multicenter comparative study in Korea indicated that RDG has few advantages compared with LDG regarding short-term surgical outcomes (24).

In order to establish the value of RDG as a treatment for gastric cancer, it must be confirmed that it is less-invasive and has similar operative quality to LDG. In our results, the number of harvested lymph nodes in RDG was similar to or rather more than that obtained with LDG, indicating that the oncological efficacy of RDG was not inferior to that of LDG. Recently, Obama et al. reported that long-term outcomes were not different between RDG and LDG (25). Nevertheless, the operative wound, extent of lymph node dissection, and surgical procedure for RDG must be the same as for LDG. Notably, the operative time was significantly longer for RDG than with LDG, in our study. However, better results were observed in RDG regarding blood loss, inflammatory findings, and postoperative complications. The effectiveness of RDG may be a result of the flexible robotic surgical instruments, the three-dimensional magnified view, and tremor filtering. The lower drain amylase levels 
following RDG that we saw in the present study, and which were likely secondary to less pancreatic compression, support the use of RDG; a lower drain amylase level means less potential for pancreatic fistula $(26,27)$. These findings also indicate that RDG is less invasiveness compared with LDG.

Patient QOL following RDG has not been previously reported, to our knowledge. In the present study, we evaluated post-gastrectomy syndrome and patients' QOL following RDG using the PGSAS-37 questionnaire. Patients' living status and QOL scores 1 month after RDG were better compared with scores at the same time point after LDG, when the surgical procedure was identical regarding the extent of gastrectomy, lymph node dissection, and the reconstruction methods. The improved scores after RDG likely reflected the lower complication rate and milder inflammatory response. Furthermore, the less-invasive characteristics of RDG are likely linked to the early recovery of gastrointestinal motility, digestion, and absorption, which results in much milder esophageal reflux and better living status.

The following limitations must be acknowledged for the present study: Firstly, all patient data were retrospectively collected and were not randomized; therefore, selection bias may be present. Secondly, the initial consecutive 21 patients were enrolled in the RDG group, and the operative time for RDG was significantly longer than that for LDG; however, we believe that surgical quality was maintained based on finding non-inferior blood loss, higher number of harvested lymph nodes, and lower incidence of complications following RDG. Longer operative times can be improved with more surgical experience. Thirdly, in addition to the single-institution design, our sample size was small, especially for the RDG group, because the use of the da Vinci surgical system was limited at our hospital. Fourthly, RDG was performed at the patient's expense until December 2019, while LDG was covered by Japan's national health insurance system. Based on these limitations, the statistical power of our study was too low for its findings to be conclusive. A prospective, randomized, large-scale study is required to establish the effectiveness of robotic surgery for gastric cancer.

In conclusion, our results suggest that RDG is a feasible, safe, and highly satisfactory technique for early gastric cancer. In addition, RDG may be associated with fewer postoperative complications including pancreatic fistula, although certain problems must be addressed, namely, the long operative time, high cost, and limited experience. We believe that effective use of robotic surgery systems will enable safe and precise minimally invasive surgery.

\section{Acknowledgements}

The Authors thank Jane Charbonneau, DVM, from Edanz Group (www.edanzediting.com/ac) for editing a draft of this article. This research did not receive any specific grant from funding agencies in the public, commercial, or not-for-profit sectors.

\section{Conflices of Interest}

All Authors have no conflicts of interest to declare.

\section{References}

1 Kitano S, Yasuda K, Shiraishi N: Laparoscopic surgical resection for early gastric cancer. Eur J Gastroenterol Hepatol 40(9): 855861, 2006. PMID: 17016135.

2 Sakuramoto S, Kikuchi S, Kuroyama S, Futawatari N, Katada $\mathrm{N}$, Kobayashi N and Watanabe M: Laparoscopy-assisted distal gastrectomy for early gastric cancer: experience with 111 consecutive patients. Surg Endosc 20(1): 55-60, 2006. PMID: 16283580. DOI: $10.1007 / \mathrm{s} 00464-005-0126-5$

3 Katai H, Sasako M, Fukuda H, Nakamura K, Hiki N, Saka M, Yamaue H, Yoshikawa T, Kojima K; JCOG Gastric Cancer Surgical Study Group: Safety and feasibility of laparoscopyassisted distal gastrectomy with suprapancreatic nodal dissection for clinical stage I gastric cancer: A multicenter phase II trial (JCOG 0703). Gastric Cancer 13(4): 238-244, 2010. PMID: 21128059. DOI: $10.1007 / \mathrm{s} 10120-010-0565-0$

4 Hiki N, Honda M, Etoh T, Yoshida K, Kodera Y, Kakeji Y, Kumamaru H, Miyata H, Yamashita Y, Inomata M, Konno H, Seto $\mathrm{Y}$ and Kitano S: Higher incidence of pancreatic fistula in laparoscopic gastrectomy. Real-world evidence from a nationwide prospective cohort study. Gastric Cancer 21(1): 162-170, 2018. PMID: 28887712. DOI: 10.1007/s10120-0170764-z

5 Ida S, Hiki N, Ishizawa T, Kuriki Y, Kamiya M, Urano Y, Nakamura T, Tsuda Y, Kano Y, Kumagai K, Nunobe S, Ohashi $\mathrm{M}$ and Sano T: Pancreatic compression during lymph node dissection in laparoscopic gastrectomy: Possible cause of pancreatic leakage. J Gastric Cancer 18(2): 134-141, 2018. PMID: 29984063. DOI: 10.5230/jgc.2018.18.e15

6 Son T and Hyung WJ: Robotic gastrectomy for gastric cancer. J Surg Oncol 112(3): 271-278, 2015. PMID: 26031408.

7 Marano A, Choi YY, Hyung WJ, Kim YM, Kim J and Noh SH: Robotic versus laparoscopic versus open gastrectomy: A metaanalysis. J Gastric Cancer 13(3): 136-418, 2013. PMID: 24156033. DOI: $10.5230 /$ jgc.2013.13.3.136

8 Song J, Kang WH, Oh SJ, Hyung WJ, Choi SH and Noh SH: Role of robotic gastrectomy using da Vinci system compared with laparoscopic gastrectomy: initial experience of 20 consecutive cases. Surg Endosc 23(6): 1204-1211, 2009. PMID: 19263147. DOI: $10.1007 / \mathrm{s} 00464-009-0351-4$

9 Song J, Oh SJ, Kang WH, Hyung WJ, Choi SH and Noh SH: Robot-assisted gastrectomy with lymph node dissection for gastric cancer: lessons learned from an initial 100 consecutive procedures. Ann Surg 249(6): 927-932, 2009. PMID: 19474671. DOI: 10.1097/01.sla.0000351688.64999.73

10 Woo Y, Hyung WJ, Pak KH, Inaba K, Obama K, Choi SH, Noh SH Robotic gastrectomy as an oncologically sound alternative to laparoscopic resections for the treatment of early-stage gastric cancers. Arch Surg 146(9): 1086-1092, 2011. PMID: 21576595. DOI: $10.1001 /$ archsurg.2011.114

11 Yang SY, Roh KH, Kim YN, Cho M, Lim SH, Son T, Hyung WJ and Kim HI: Surgical outcomes after open, laparoscopic and robotic gastrectomy for gastric cancer. Ann Surg Oncol 24: 1770-1777, 2017. 
12 Uyama I, Suda K, Nakauchi M, Kinoshita T, Noshiro H, Takiguchi S, Ehara K, Obama K, Kuwabara S, Okabe H and Terashima M: Clinical advantages of robotic gastrectomy for clinical stage I/II gastric cancer: A multi-institutional prospective single-arm study. Gastric Cancer 22(2): 377-385, 2019. PMID: 30506394. DOI: $10.1007 / \mathrm{s} 10120-018-00906-8$

13 Japanese Gastric Cancer Association. Japanese Classification of Gastric Carcinoma: 3rd English Edition. Gastric Cancer 14(2): 101112, 2011. PMID: 21573743. DOI: 10.1007/s10120-011-0041-5

14 Persson PB: Good publication practice in physiology 2017: Current Revisions of the Recommendations for the Conduct, Reporting, Editing and Publication of Scholarly Work in Medical Journals. Acta Physiol 221(4): 283-284, 2017. DOI: 10.1111/ apha.12984

15 Japanese Gastric Cancer Association. Japanese Gastric Cancer Treatment Guidelines, v1; 2018.

16 Dindo D, Demartines $\mathrm{N}$ and Clavien PA: Classification of surgical complications: a new proposal with evaluation in a cohort of 6336 patients and results of a survey. Ann Surg 240(2): 205-213, 2004. PMID: 1527354. DOI: 10.1097/01.sla.000013 3083.54934.ae

17 Clavien PA, Barkun J, de Oliveira ML, Vauthey JN, Dindo D, Schulick RD, de Santibañes E, Pekolj J, Slankamenac K and Bassi C: The Clavien-Dindo classification of surgical complications: Five-year experience. Ann Surg 250(2): 187-196, 2009. PMID: 19638912. DOI: 10.1097/SLA.0b0 13e3181b13ca2

18 Nakada K, Ikeda M, Takahashi M, Kinami S, Yoshida M, Uenosono Y, Kawashima Y, Oshio A, Suzukamo Y, Terashima $\mathrm{M}$ and Kodera $\mathrm{Y}$ : Characteristics and clinical relevance of postgastrectomy syndrome assessment scale (PGSAS)-45: newly developed integrated questionnaires for assessment of living status and quality of life in postgastrectomy patients. Gastric Cancer 18(1): 147-58, 2015. PMID: 24515247. DOI: 10.1007/ s10120-014-0344-4

19 Crew KD and Neugut AI: Epidemiology of gastric cancer. World J Gastroenterol 12(3): 354-362, 2006. PMID: 16489633 DOI: 10.3748/wjg.v12.i3.354

20 Shiraishi N, Yasuda K and Kitano S: Laparoscopic gastrectomy with lymph node dissection for gastric cancer. Gastric Cancer 9(3): 167-176, 2006. PMID: 16952034. DOI: 10.1007/s10120006-0380-9
21 Kitano S, Iso Y, Moriyama M and Sugimachi K: Laparoscopyassisted Billroth I gastrectomy. Surg Laparosc Endosc 4(2): 146148, 1994. PMID: 8180768.

22 14th Nationwide Survey of Endoscopic Surgery in Japan. J Jpn Soc Endosc Surg 23: 773-778, 2018 (in Japanese).

23 Suda K, Man-I M, Ishida Y, Kawamura Y, Satoh, Uyama.I: Potential advantages of robotic radical gastrectomy for gastric adenocarcinoma in comparison with conventional laparoscopic approach: A single institutional retrospective comparative cohort study. Surg Endosc 29(3): 673-685, 2015. PMID: 25030478. DOI: $10.1007 / \mathrm{s} 00464-014-3718-0$

24 Kim HI, Han SU, Yang HK, Kim YW, Lee HJ, Ryu KW, Park JM, An JY, Kim MC, Park S, Song KY, Oh SJ, Kong SH, Suh BJ, Yang DH, Ha TK, Kim YN and Hyung WJ: Multicenter prospective comparative study of robotic versus laparoscopic gastrectomy for gastric adenocarcinoma. Ann Surg 263(1): 103109, 2016. PMID: 26020107. DOI: 10.1097/SLA.000000 0000001249

25 Obama K, Kim YM, Kang DR, Son T, Kim HI, Noh SH and Hyung WJ: Long-term oncologic outcomes of robotic gastrectomy for gastric cancer compared with laparoscopic gastrectomy. Gastric Cancer 21(2): 285-295, 2018. PMID: 28639136 DOI: $10.1007 / \mathrm{s} 10120-017-0740-7$

26 Obama K, Okabe H, Hosogi H, Tanaka E, Itami A and Sakai Y: Feasibility of laparoscopic gastrectomy with radical lymph node dissection for gastric cancer: from a viewpoint of pancreasrelated complications. Surgery 149(1): 15-21, 2011. PMID: 20627337. DOI: 10.1016/j.surg.2010.04.014

27 De Sol A, Cirocchi R, Di Patrizi MS, Boccolini A, Barillaro I, Cacurri A, Grassi V, Corsi A, Renzi C, Giuliani D, Coccetta M and Avenia N: The measurement of amylase in drain fluid for the detection of pancreatic fistula after gastric cancer surgery: an interim analysis. World J Surg Oncol 13: 65, 2015. PMID: 25849316. DOI: 10.1186/s12957-014-0428-y 